\title{
Growth Assisted by Glancing Angle Deposition: A New Technique to Fabricate Highly Porous Anisotropic Thin Films
}

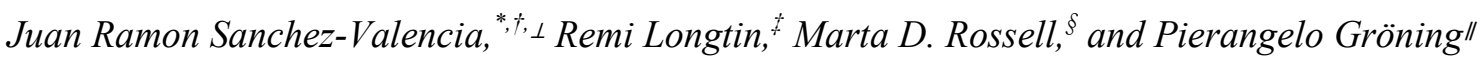 \\ †nanotech@surfaces Laboratory, Joining Technologies and Corrosion Laboratory, ${ }^{\S}$ Electron \\ Microscopy Center, and \|Department of Advanced Materials and Surfaces, Empa, Swiss \\ Federal Laboratories for Materials Science and Technology, Ueberlandstrasse 129,
}

CH-8600 Duebendorf, Switzerland

${ }^{\perp}$ present address: Nanotechnology on Surfaces Lab. Institute of Materials Science of Seville. C/ Americo Vespucio 49, 41092, Seville, Spain

Corresponding Author: * e-mail: jrsanchez@icmse.csic.es

KEYWORDS: GLAD, GAGLAD, hybrid materials, anisotropy, porous thin films

\begin{abstract}
We report a new methodology based on glancing angle deposition (GLAD) of an organic molecule in combination with perpendicular growth of a second inorganic material. The resulting thin films retain a very well-defined tilted columnar microstructure characteristic of GLAD with the inorganic material embedded inside the columns. We refer to this new methodology as growth assisted by glancing angle deposition or GAGLAD, since the material of interest (here, the inorganic) grows in the form of tilted columns, though it is deposited under a non-glancing configuration. As a "proof of concept", we have used silver and zinc oxide as the perpendicularly deposited material since they usually form ill-defined columnar microstructures at room temperature by GLAD. By means of our GAGLAD methodology, the typical tilted columnar microstructure can be developed for materials that otherwise do not form ordered structures under conventional GLAD. This simple methodology broadens significantly the range of materials where control of the microstructure can be achieved by tuning the geometrical deposition parameters. The two examples presented here, Ag/Alq3 and $\mathrm{ZnO} / \mathrm{Alq} 3$, have been deposited by physical vapor deposition (PVD) and plasma enhanced chemical vapor deposition (PECVD), respectively: two different vacuum techniques that illustrate the generality of the proposed technique. The two type of hybrid samples present very interesting properties that demonstrate the potentiality of GAGLAD. On one hand, the Ag/Alq3 samples present highly optical anisotropic properties when they are analyzed with linearly polarized light. To our knowledge, these $\mathrm{Ag} / \mathrm{Alq} 3$ samples present the highest angular selectivity reported in the visible range. On the other hand, $\mathrm{ZnO} / \mathrm{Alq} 3$ samples are used to develop highly porous $\mathrm{ZnO}$ thin films by using Alq3 as sacrificial material. In this way, antireflective $\mathrm{ZnO}$ samples with very low refractive index and extinction coefficient have been obtained.
\end{abstract}

This document is the accepted manuscript version of the following article: Sanchez-Valencia, J. R., Longtin, R., Rosse11, M. D., \& Gröning, P. (2016). Growth assisted by glancing angle deposition: a new technique to fabricate highty porous anisotropic thin films. ACS Applied Materials and Interfaces, 8(13), 8686-8693. http://doi.org/10.1021/acsami . 6b00232 


\section{INTRODUCTION}

Glancing angle deposition (GLAD) is a very well established technique to engineer nanostructures by controlling different geometric parameters during deposition. ${ }^{1-3}$ The singular tilted nanocolumnar morphology that can be obtained is caused by a self-shadowing mechanism provoked by the oblique incidence of evaporated molecules with respect to the substrate. Thin films obtained by GLAD possess a high open porosity and have been used for development of optical sensors and fluorescent devices. ${ }^{4-8}$

Fabrication of ordered nanostructures by GLAD has a strong dependency on the nature of the material. ${ }^{9-12}$ For example, noble metals possess a very high surface diffusivity that hampers proper columnar growth at room temperature and usually results in ill-defined columns that broaden with film thickness. ${ }^{13-15}$ Other materials like $\mathrm{ZnO}$ are not suitable for thermal or electronic evaporation due to chemical reduction even under ultrahigh vacuum (UHV) conditions. ${ }^{16,17}$ In the latter case, other techniques like magnetron sputtering can be used, but this reduces the collimation of material flux during evaporation and therefore produces less defined nanocolumns.

On the other hand, GLAD deposition of organic molecules is gaining importance to develop highly ordered nanostructures for numerous applications in fields such as solar cells or fluorescent optical devices. ${ }^{18-21}$ Brett and co-workers ${ }^{18}$ reported the fabrication of luminescent organic chiral thin films by using a well-known molecule for the fabrication of organic lightemitting diodes (OLED), tri(8-hydroxyquinoline) aluminum (Alq3). ${ }^{19}$

In this work, we have taken advantage of GLAD of Alq3 molecules in order to produce highly uniform and ordered hybrid organic-inorganic nanostructures. ${ }^{18,19}$ GLAD of Alq3 has been performed in combination with a remote electron cyclotron resonance (ECR) plasma source working at very low pressures (around $10^{-4} \mathrm{mbar}$ ). Such low pressure is needed to maintain directionality of the molecular flux during glancing angle deposition. At the same time, the energetic and reactive species in the plasma were used to deposit an additional inorganic material. Codeposition of more than one material, all of them under oblique conditions either simultaneously ${ }^{22-29}$ or in the form of multilayers ${ }^{30-32}$ has been reported in the literature. The essential difference of our work with respect to standard GLAD and codeposition techniques (usually referred as $\mathrm{GLACD}^{25}$ ) is that here the inorganic material is deposited at a perpendicular geometry with respect to the substrate while GLAD of a second material (in our case, organic) is taking place simultaneously. For this reason, we refer to this new deposition method as growth assisted by glancing angle deposition or GAGLAD. A scheme of the deposition technique is shown in Figure 1). The basic idea is to combine GLAD of materials or molecules like Alq3, which form ordered and porous nanostructures, with nonglancing deposition of a second material, namely inorganic, which otherwise does not form ordered structures under standard GLAD. The resulting hybrid nanostructured films have the functionalities of GLAD, such as having a very porous tilted columnar microstructure, even when the inorganic counterpart would not form this microstructure by its own. The usefulness of this technique will be illustrated with two materials grown by different techniques: silver by physical vapor deposition (PVD) and zinc oxide by plasma enhanced chemical vapor deposition (PECVD), 
both in combination with Alq3. Although in this work we have employed a plasma to deposit the inorganic material, the GAGLAD technique proposed here does not require it.

\section{EXPERIMENTAL SECTION}

Materials. Tris(8-hydroxyquinoline)aluminum (Alq3) and diethylzinc $\left(\mathrm{Et}_{2} \mathrm{Zn}\right)$ were supplied by Sigma-Aldrich and used without further purification. Silver wire (99.99\% purity) of $0.25 \mathrm{~mm}$ diameter was acquired from Goodfellow.

Sample Preparation. The high-vacuum deposition system employed consists of a plasma reactor working in a downstream configuration. The plasma is operated with an external microwave source working at a power of $200 \mathrm{~W}$ coupled through a flat pyrex window. The reactor possesses a set of external magnets to work in electron cyclotron resonance (ECR), sustaining the plasma at very low pressures (on the order of $10^{-4} \mathrm{mbar}$ ). The system reaches a base pressure of $2 \times 10^{-7}$ mbar by means of a turbo molecular pump. Alq3 molecules were evaporated in a Knudsen-type cell at around $240{ }^{\circ} \mathrm{C}$. The temperature of the evaporator was adjusted to obtain a constant deposition rate, measured during the deposition with a quartz cristal monitor (QCM) acquired from Inficon (SQM-160) placed at the same distance from the samples but with a perpendicular configuration (the thickness monitored is then much higher). Samples were located at $12 \mathrm{~cm}$ from the evaporator, with a glancing angle of $80^{\circ}$ with respect to the evaporated molecules. A sketch of the experimental setup is shown in Figure 1. Substrates of ca. $1.5 \times 2 \mathrm{~cm}^{2}$ were placed horizontally to ensure a similar distance to the evaporator. With these experimental conditions, only two substrates were grown at a time but a homogeneous deposition is warranted. Argon, oxygen, and diethylzinc were dosed by using a precision leak valve. $\mathrm{Si}(100)$ wafers and fused silica substrates were used to measure scanning electron microscopy (SEM) and optical spectra, respectively.

The silver wire used for preparation of hybrid Ag/Alq3 samples was located $4 \mathrm{~cm}$ from the samples and biased at $-450 \mathrm{~V}$. The pressure during the deposition was set to $1 \times 10^{-4}$ mbar by dosing Ar into the chamber. While the ECR microwave plasma is off, the current monitored at the wire was 0 regardless of the bias voltage, and no glow discharge could be observed at the wire. By contrast, when the ECR plasma is turned on, the generated ions are accelerated toward the wire, recording a current of $2 \mathrm{~mA}$ (at $-450 \mathrm{~V}$ ), and a glow discharge over the wire could be observed. The deposition rate of silver was constant while the rate of Alq3 molecules was controlled to 2, 4, and $8 \AA / \mathrm{s}$ as measured in the QCM. The deposition time was adjusted in order to obtain a fixed amount of Alq3 $(1 \mu \mathrm{m})$ and, consequently, obtaining different amounts of Ag. The equivalent thickness of silver for each sample was $50 \mathrm{~nm}(8 \AA / \mathrm{s}), 100 \mathrm{~nm}(4 \AA / \mathrm{s})$, and 200 $\mathrm{nm}(2 \AA / \mathrm{s})$, determined from SEM examination of the film cross sections in the absence of organic molecules (see Figure S1). The drawback of this methodology is that plasma interaction with the sample is higher/ lower for the lower/higher deposition rates of Alq3, thus having an important contribution in the thin-film microstructure.

For preparation of the hybrid $\mathrm{ZnO} / \mathrm{Alq} 3$ samples, the precursor $\mathrm{Et}_{2} \mathrm{Zn}$ and oxygen were dosed with the same flow, ${ }^{33}$ and the pressure was $5 \times 10^{-4}$ mbar during deposition. For this sample, the QCM rate of Alq3 was adjusted to $4 \AA / \mathrm{s}$ and a total thickness of $1 \mu \mathrm{m}$ was deposited. Annealing of the samples was performed on a "hot plate" at $550{ }^{\circ} \mathrm{C}$ for $15 \mathrm{~min}$. 
Charaterization Methods. Hybrid samples were examined by field emission scanning electron microscopy on a FEI NovaNanoSEM 230. Cross-sectional views were obtained by cleaving the samples deposited on $\mathrm{Si}(100)$. Compositional analysis was performed in the same microscope by means of a energy-dispersive X-ray spectroscopy (EDX) detector (X-Max, Oxford Instruments), using $20 \mathrm{kV}$ of acceleration voltage.

Samples for tomography were prepared by scratching the film with a razor blade and dispersing the resulting powder in ethanol. Subsequently, one drop of the dispersion containing Ag/Alq3 columns was deposited onto a carbon-coated copper grid and dried in air at $60{ }^{\circ} \mathrm{C}$. Annular dark-field scanning transmission electron microscopy (ADF-STEM) and tomography were performed on a JEOL 2200FS TEM/STEM operated at $200 \mathrm{kV}$ and equipped with a Gatan Digiscan system. A JEOL single-axis tomography holder was used, and ADFSTEM images were manually recorded at $2^{\circ}$ tilt intervals over a range from $-72^{\circ}$ to $+66^{\circ}$. The 70 images were aligned by use of the StackReg plugin for the image processing software ImageJ. Threedimensional (3D) volume reconstruction was computed with 50 cycles of the simultaneous iterative reconstruction technique (SIRT) implemented in TomoJ software, which is also an ImageJ plugin. Finally, voxel rendering and slice viewing were generated with the Amira visualization program.

UV-vis transmittance spectra were recorded in a Cary 50 (Varian) spectrophotometer. For polarization measurements, a Glan-Taylor prism acquired from Edmund Optics was used.

The refractive index of the $\mathrm{ZnO} / \mathrm{Alq} 3$ sample annealed at $550{ }^{\circ} \mathrm{C}$ in air was extracted from the UV-vis transmittance spectrum, which consists of an interference pattern of the wave fronts of the incoming beam and the partially reflected beams at the film-substrate and film-air interfaces. Due to the low refractive index, the interference is very weak and no oscillations were observed. A nonlinear curve fit was performed by using the software Origin 8 (OriginLab Corp.). The complex refractive index dispersion was taken as:

$$
\widetilde{n}=n+i k\left\{\begin{array}{c}
n(\lambda)=n_{0}+\left(\frac{\lambda_{0}}{\lambda}\right)^{2} \\
k(\lambda)=\left(\frac{k_{d}}{\lambda}\right)^{4}
\end{array}\right.
$$

where $\mathrm{n}_{0}, \lambda_{0}$, and $\mathrm{k}_{\mathrm{d}}$ are fitting parameters. The imaginary part of the refractive index was assumed to follow a Rayleigh dispersion law (fourth inverse power).

The porosity of the $\mathrm{ZnO} / \mathrm{Alq} 3$ samples annealed in air at $550{ }^{\circ} \mathrm{C}$ was evaluated by means of the effective medium theory, concretely the Bruggeman model. The model follows the equation:

$$
\frac{(1-\mathrm{P})}{100} \frac{\mathrm{n}_{\text {ox }}^{2}-\mathrm{n}_{\text {eff }}^{2}}{\mathrm{n}_{\text {ox }}^{2}+2 \mathrm{n}_{\text {eff }}^{2}}+\frac{\mathrm{P}}{100} \frac{\mathrm{n}_{\text {air }}^{2}-\mathrm{n}_{\text {eff }}^{2}}{n_{\text {air }}^{2}+2 \mathrm{n}_{\text {eff }}^{2}}=0
$$

where $\mathrm{P}$ is the porosity as a percentage, nox is the refractive index of the bulk oxide $\left[\mathrm{n}_{\mathrm{ZnO}}(550\right.$ $\mathrm{nm})=2.02$ and $\left.\mathrm{n}_{\mathrm{Al} 203}(550 \mathrm{~nm})=1.77\right], \mathrm{n}_{\text {air }}=1$ (refractive index of air), and $\mathrm{n}_{\text {eff }}$ is the effective refractive index, in our case the one extracted from the UV-vis spectrum. 


\section{RESULTS AND DISCUSSION}

Ag/Alq3 Hybrid GAGLAD Thin Films. In our first example, argon plasma in combination with a negatively biased silver wire was used. $\mathrm{The}^{+}{ }^{+}$ions from the plasma are then accelerated toward the wire, producing the sputtering of silver atoms that are then redeposited over the substrates at a perpendicular geometry (see Figure 1).

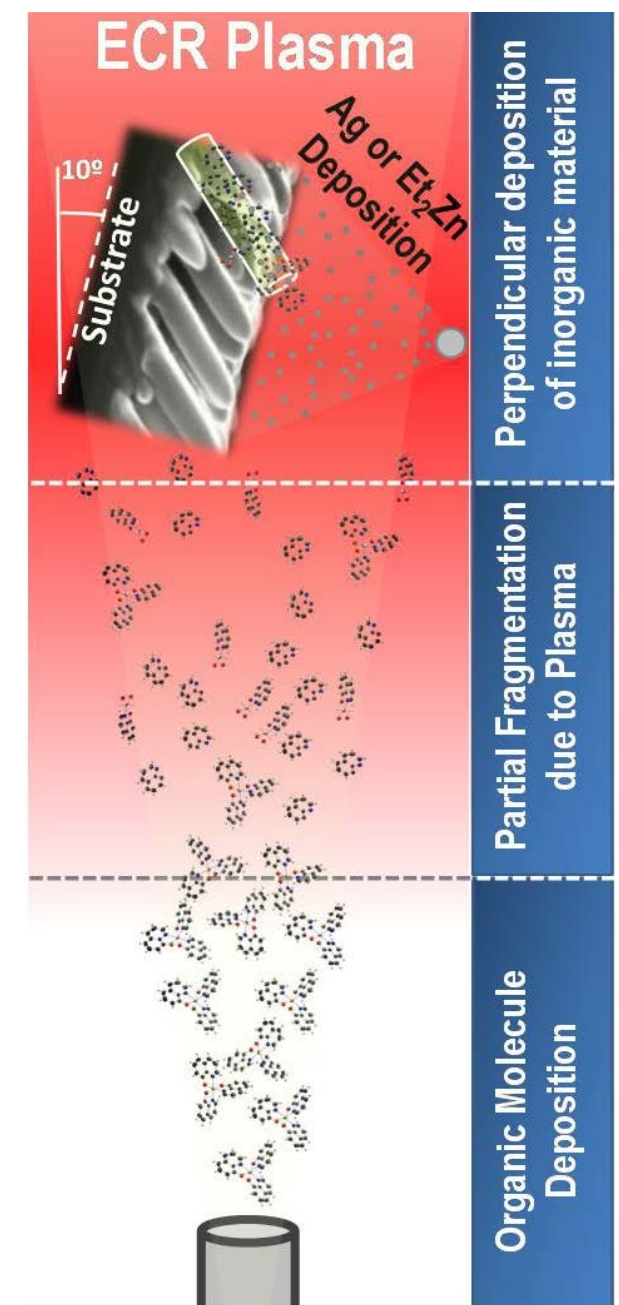

Figure 1. Scheme of experimental setup used for GAGLAD.

Figure 2 shows cross-sectional $(a-d)$ and top-view $(e-h)$ SEM pictures of the three hybrid Ag/Alq3 samples prepared. The equivalent thickness of silver for each sample was 50 (a, e), 100 (b, f), and $200 \mathrm{~nm}$ (c, d, g, h), determined from SEM examination of film cross sections in the absence of organic molecules (see Figure S1). As can be observed in Figure 2, the nanostructure of the samples is completely modified by simply changing the amount of $\mathrm{Ag}$ and the plasma interaction during deposition. The differences observed in thickness cannot be described only by the excess of silver between the samples $(200$ and $50 \mathrm{~nm}$ for maximum and minimum, respectively): they can only be explained by an increase in porosity. This increase can be identified in the cross-sectional SEM pictures of Figure $2 \mathrm{a}-\mathrm{c}$, where intercolumnar separation along the y-direction increases with the amount of silver from around 40-50 to 80-100 nm for minimum and maximum amount, respectively. At the same time, the size of the columns along 
the $y$-direction remains almost unaltered (see Figure $2 a-c$ ), while along the $x$-axis (see top-view pictures, Figure $2 \mathrm{e}-\mathrm{h}$ ), their size increases with amount of Ag. The increase in size along the $\mathrm{x}-$ direction is due to two factors: first, the nanocolumns coalesce due to a bundling effect that occurs in the direction perpendicular to the molecular flux during growth. ${ }^{1,34-36}$ Second, this reduced separation produces fusion of the columns, likely due to partial fragmentation of the molecules because of the plasma, giving rise to anisotropic nanocolumns. This effect can be observed in the top views and more easily in the higher magnification SEM pictures of Figure $2 \mathrm{~h}$.

Another remarkable feature of the microstructure observed is the tilting angle of the columns. The inclination of the columns decreases with Ag amount/plasma interaction. This behavior has been observed by Parra-Barranco et al. ${ }^{37}$ in the plasma-assisted deposition of indium tin oxide (ITO) by GLAD. The ITO samples grown under plasma presented a smaller tilting angle of the columns together with increased nanocolumnar separation. The agreement with these results indicates that plasma interaction during growth is likely responsible for the increase in porosity and the reduction of tilting angle. Despite the lack of literature about plasma-assisted growth under GLAD, it is clear that the plasma modifies the columnar shadowing and an additional model different from the tangent rule needs to be applied. ${ }^{9-11}$ Alvarez et al. ${ }^{12}$ have recently reported a model that takes into consideration modification of shadowing near column tips due to a "surface trapping mechanism". This mechanism modifies the trajectory of the evaporated molecules due to short-range interactions (below 3-4 $\AA$ ) such as electrostatic or van der Waals, diminishing the tilting angle at the same time that the columnar separation increases. This mechanism depends exclusively on the material deposited but can be applied here since the formation of radicals due to the plasma produces reactive sites that can lead to an increase in these short-range interactions. In any case, it is important to outline that the porosity can be tuned by using the GAGLAD technique proposed here.

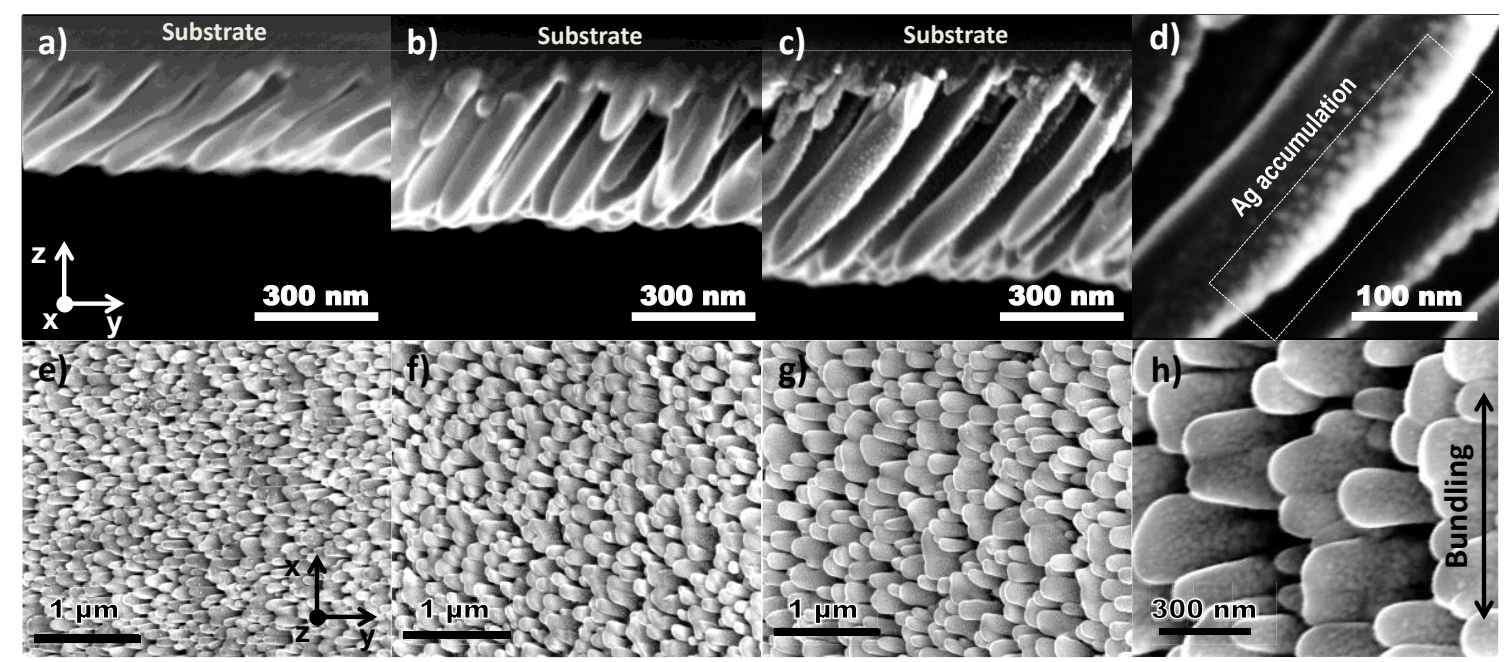

Figure 2. Cross-sectional $(\mathrm{a}-\mathrm{d})$ and top-view $(\mathrm{e}-\mathrm{h}) \mathrm{SEM}$ pictures of $\mathrm{Ag} / \mathrm{Alq} 3$ hybrid samples with fixed amount of Alq3 and variable amounts of silver: $50 \mathrm{~nm}(\mathrm{a}, \mathrm{e}), 100 \mathrm{~nm}(\mathrm{~b}, \mathrm{f})$, or 200 (c, d, g, h) of equivalent thickness. $(\mathrm{d}, \mathrm{h})$ Higher magnification pictures of panels $\mathrm{c}$ and $\mathrm{g}$, respectively. 
Distribution of the inorganic counterpart within the columns is very important for the final application of the films. From the cross-sectional SEM pictures (particularly Figure 2d), it can be observed that silver is preferentially located along the lateral side facing the silver wire (a higher accumulation of $\mathrm{Ag}$ is observed with a higher contrast). We performed electron tomography using annular dark-field scanning transmission electron microscopy (ADF-STEM) on a sample with an intermediate amount of $\mathrm{Ag}(100 \mathrm{~nm})$ in order to reveal the spatial distribution of Ag nanoparticles inside the Alq3 columns.

Figure 3a presents an ADF-STEM image of the tomographic tilt series showing two joined columns (the joining direction coincides with the x-axis) fully covered with Ag nanoparticles (see also Movie S1). The 3D tomographic reconstruction reveals that the nanoparticles are preferentially located on the surface of Alq3 columns. In Figure 3b, a voxel projection along the electron beam direction is shown. However, in order to assess the structure of these hybrid nanostructures. A sub-volume, indicated with four white arrows in Figure 3b, was extracted and is represented in Figure 3c. The subvolume rendering of Figure $3 \mathrm{~b}$ unambiguously proves the presence of nanoparticles on both sides of the Alq3 columns, which are seen to be slightly curved. Nanoparticles located on the concave (left) side of the columns are very small (from 2 to $7 \mathrm{~nm}$ ), while on the convex (right) side of the columns the Ag amount is much higher, and the nanoparticles are very inhomogeneous, with sizes ranging from $2 \mathrm{~nm}$ to several tens of nanometers. A size distribution study of the silver nanoparticles does not reveal any remarkable conclusion due to the lack in homogeneity, anisotropic shapes, and partial coalescence. To visualize the nanoparticles, Figure $3 \mathrm{~d}-\mathrm{g}$ presents four orthoslices at different positions through the 3D volume reconstruction. These vertical orthoslices further demonstrate the presence of $\mathrm{Ag}$ nanoparticles on both sides of the columns. Larger coverage is distinctly observed for the side facing the silver wire during the film growth (Figure $3 \mathrm{~g}$ ). A movie of the full series of orthoslices is shown in Movie S2. 

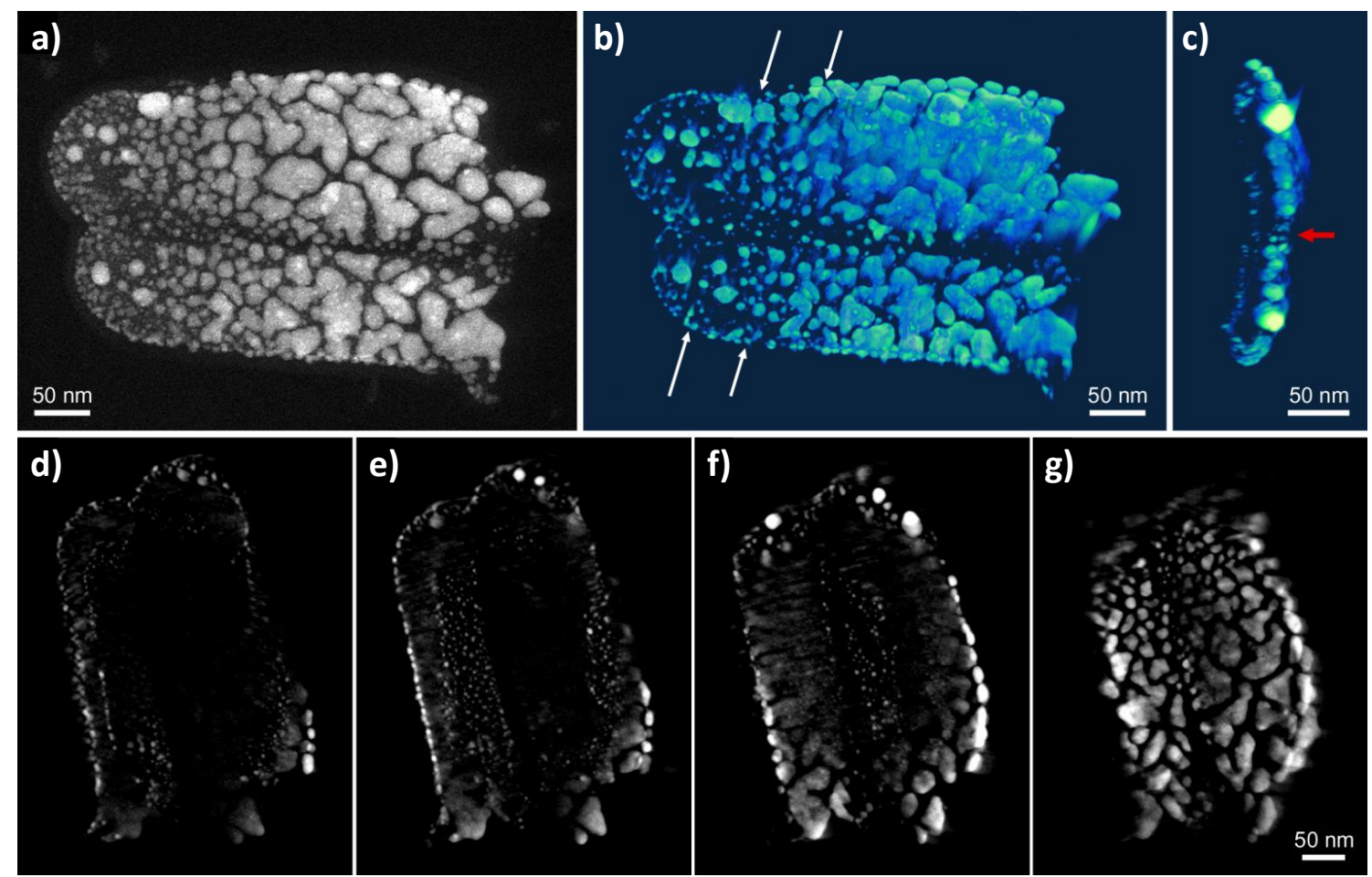

Figure 3. (a) ADF-STEM image of two joined columns corresponding to the Ag/Alq3 hybrid sample with $100 \mathrm{~nm} \mathrm{Ag.} \mathrm{(b)} \mathrm{Voxel} \mathrm{projection} \mathrm{view} \mathrm{along} \mathrm{the} \mathrm{electron} \mathrm{beam} \mathrm{direction} \mathrm{z.} \mathrm{(c)} \mathrm{Voxel} \mathrm{projection} \mathrm{of} \mathrm{a}$ subvolume extracted from the area indicated with four arrows in panel $b$. The red arrow indicates the coalescent area between the two columns. $(\mathrm{d}-\mathrm{g})$ Orthoslices at four different positions through the 3D tomographic reconstruction.

Hybrid Ag/Alq3 nanocolumnar films possess particular optical properties originating from the different components: the Alq3 structure benefits from the molecule fluorescence (see Figure S2), while the silver nanoparticles have strong plasmonic properties. The ordered arrangement in the film is provided by Alq3, whereas the Ag nanoparticles are distributed along the ordered columns. Consequently, the optical properties for a given sample depend on the polarization of incoming light and the orientation of the thin films. For example, the samples are birefringent (present different refractive indexes along the $\mathrm{x}$ - and $\mathrm{y}$-axes) due to the Alq3 organic matrix surrounding the anisotropic nanoparticles. But the most remarkable behavior of these samples is the anisotropic absorption of light due to plasmonic properties of the metallic structures. Figure 4 presents a scheme (top) of the coordinate system chosen, where the z-axis is the propagating direction of incoming light. Figure 4 (bottom) shows the transmittance spectra for y- (black solid line) and x-polarization (black dotted line) for the samples presented in Figure 2, with (a) 50, (b) 100, and (c) $200 \mathrm{~nm} \mathrm{Ag} \mathrm{equivalent} \mathrm{thickness.} \mathrm{It} \mathrm{can} \mathrm{be} \mathrm{noted} \mathrm{that} \mathrm{the} \mathrm{transmittance} \mathrm{is}$ higher for $\mathrm{y}$ - than for $\mathrm{x}$-polarization for all samples. The mentioned bundling effect, together with fusión of the columns due to decreased distance along the $\mathrm{x}$-axis, produces stronger absorption for polarized light along this direction. ${ }^{34-36}$ At the same time, the smaller size of the columns along the y-direction induces a higher transmittance all over the spectral range investigated. 


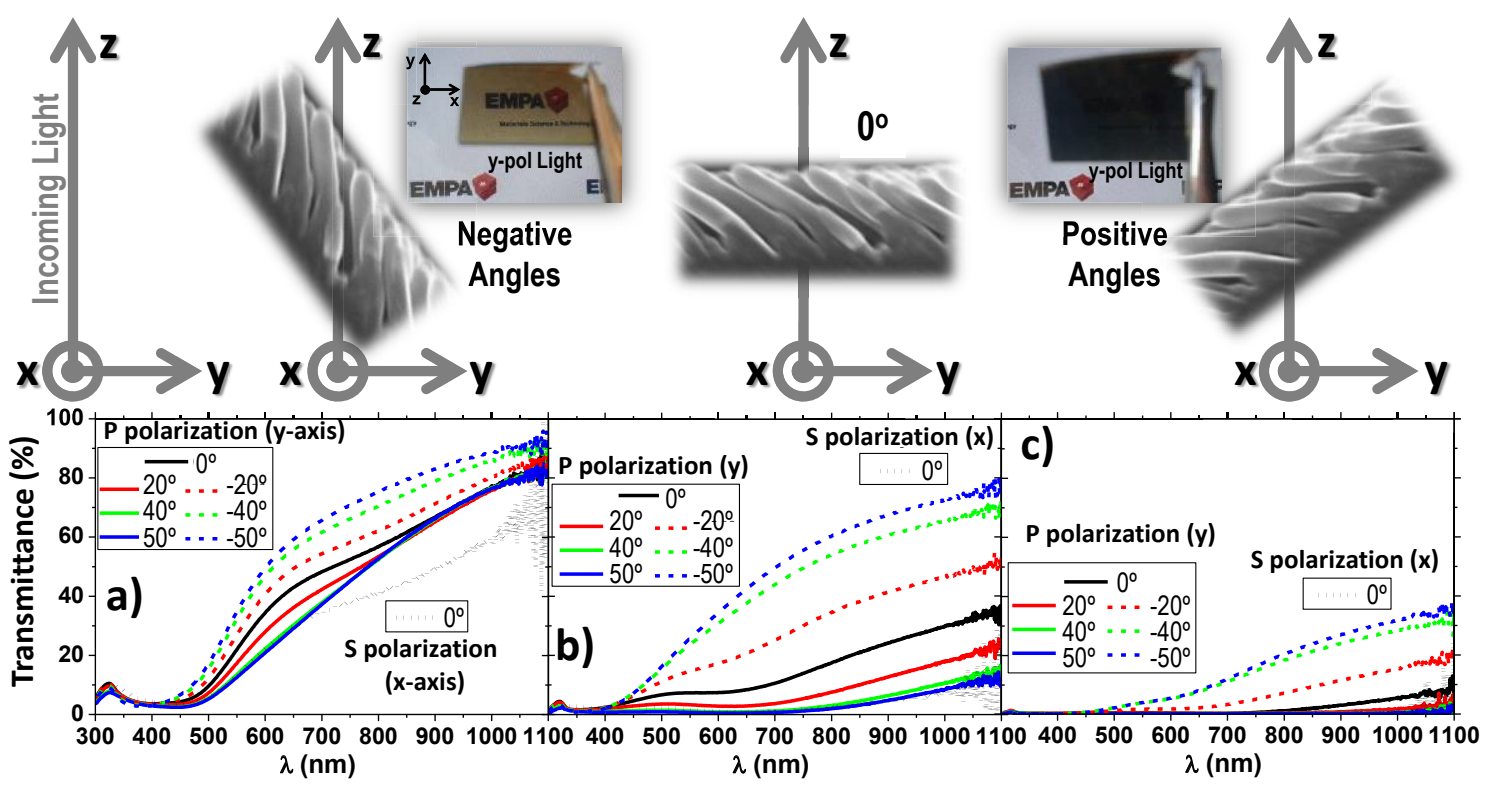

Figure 4. Transmittance spectra for y-polarized light for a fixed amount of Alq3 and (a) 50, (b) 100, and (c) $200 \mathrm{~nm} \mathrm{Ag} \mathrm{equivalent} \mathrm{thickness} \mathrm{(samples} \mathrm{in} \mathrm{Figure} \mathrm{2).} \mathrm{Samples} \mathrm{were} \mathrm{rotated} \mathrm{from}-50^{\circ}$ (dashed lines) to $+50^{\circ}$ (solid lines). The spectrum for $0^{\circ}$ and $\mathrm{x}$-polarization is also shown with dotted lines for each sample. The scheme at the top shows rotation toward negative (left) and positive (right) angles and a photograph of these two cases.

Another remarkable feature can be found in the polar rotation of the samples, which produces a drastic change in the transmittance spectra. The scheme at the top of Figure 4 shows the chosen sign for polar rotation of the samples around the $\mathrm{x}$ axis. Rotation of $20^{\circ}, 40^{\circ}$, and $50^{\circ}$ for both positive (colored solid lines) and negative angles (colored dashed lines) is presented in Figure 4 for samples with (a) 50, (b) 100, and (c) $200 \mathrm{~nm} \mathrm{Ag} \mathrm{equivalent} \mathrm{thickness.} \mathrm{A} \mathrm{large} \mathrm{change} \mathrm{in}$ transmittance spectra can be noted between negative and positive angles for p-polarized light ( $\mathrm{y}$ axis). In particular, the change in Ag/Alq3 sample with $100 \mathrm{~nm} \mathrm{Ag}$ equivalent thickness is significant in the $600-1100 \mathrm{~nm}$ range, where rotation to $-50^{\circ}$ shifts the transmittance to values higher than $50 \%$ and rotation to $+50^{\circ}$ shifts values below $10 \%$ (Figure $4 \mathrm{~b}$ ). The change in transmittance is caused by the tilted columnar structure, where rotation around the $\mathrm{x}$-axis produces an alignment of the columns (scheme in Figure 4, negative angles) or a misalignment (positive angles) with respect to incoming light. Two photographs of the sample with medium amount of $\mathrm{Ag}(100 \mathrm{~nm})$ are also shown beside the scheme for rotation around $\mathrm{x}$ axis (negative and positive angles at left and right, respectively) when the sample is illuminated with ppolarized light. In the pictures one can observe a yellowish transparent appearance for rotation toward negative angles, whereas rotation toward positive angles turns the sample opaque.

This variation in transmittance spectra between positive and negative angles is only visible for the combination of p-polarized light and rotation around the $\mathrm{x}$-axis. The p-polarized/ y-rotation, s-polarized/x-rotation, and s-polarized/y-rotation combinations do not produce any apparent change in transmittance spectra between negative and positive angles (see Figure S3) but only slight changes can be appreciated due to variation of the optical thickness. It is important to outline that the changes accounted for rotation around the x-axis can be clearly seen (although attenuated) using natural light, while rotation around the y-axis does not produce any optical 
change. This effect has been previously reported as "angular selectivity" and is related to the anisotropic geometry of embedded metallic nanoparticles in a dielectric matrix. ${ }^{38-40}$ Angularselective coatings present different transmittances for p-polarized light, that is, $\operatorname{Tp}(\theta) \neq \operatorname{Tp}(-\theta)$ for one specific polar rotation. To our knowledge, the Ag/Alq3 samples studied here depict the highest angular selectivity reported. The Ag/Alq3 with $100 \mathrm{~nm} \mathrm{Ag} \mathrm{equivalent} \mathrm{thickness} \mathrm{sample}$ possesses a maximum integrated transmittance over the spectra studied $(250-1100 \mathrm{~nm})$ of around $40 \%$ for $\theta=-50^{\circ}$ and $3 \%$ for $\theta=50^{\circ}$ for p-polarized light. Such coatings have many potential applications for energy conversion systems, privacy protection, and high signal-tonoise ratio detectors. ${ }^{41-44}$

ZnO/Alq3 Hybrid GAGLAD thin films. In our second GAGLAD example, an oxygen plasma at low pressure $(1 \times 10-4$ mbar $)$ was used to decompose the volatile organic precursor diethylzinc (Et2Zn) and deposit $\mathrm{ZnO}$ during GLAD of Alq3 molecules. Figure 5 shows (a) cross-section and (b) top-view SEM pictures of a $\mathrm{ZnO} / \mathrm{Alq} 3$ sample, in which can be observed a tilted columnar structure similar to the previous $\mathrm{Ag} / \mathrm{Alq3}$. The presence of $\mathrm{Zn}$ in the samples was verified by EDX as well as high concentrations of $\mathrm{Al}, \mathrm{C}, \mathrm{N}$, and $\mathrm{O}$ coming from Alq3 molecules.

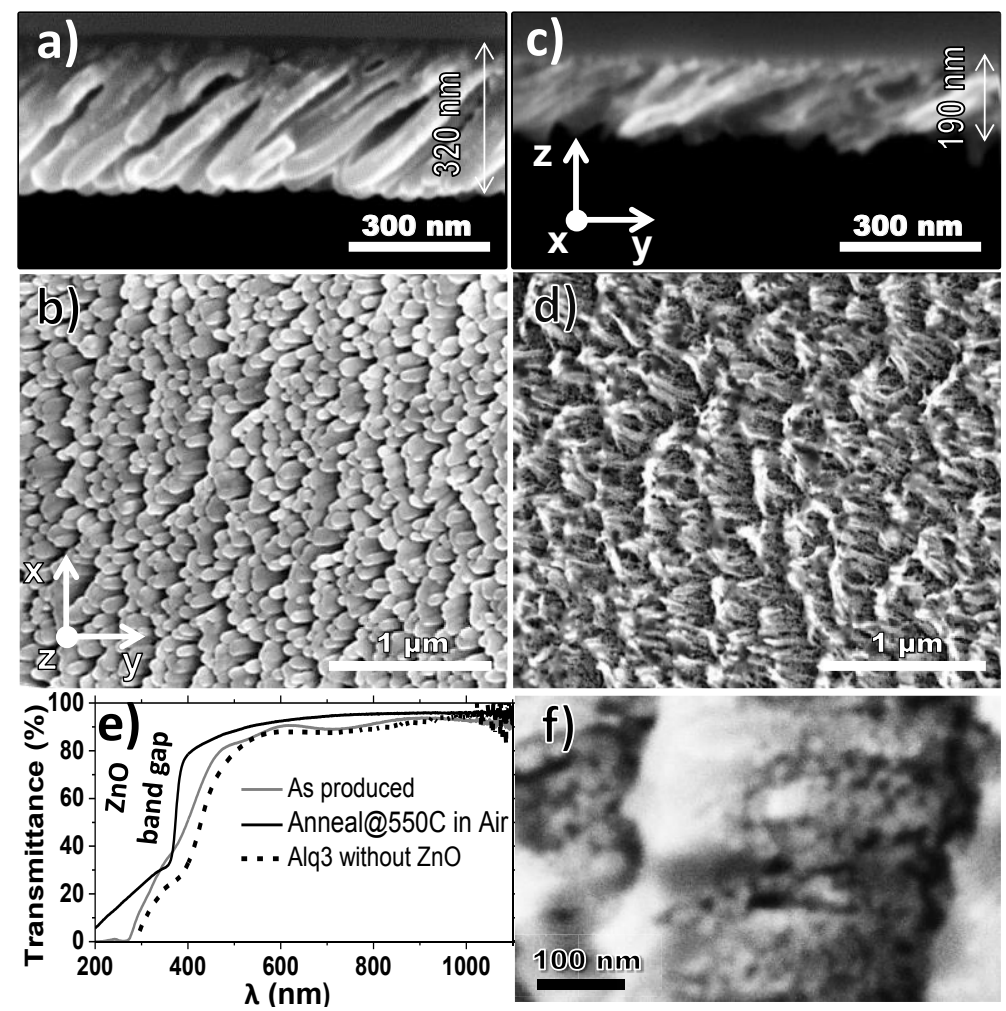

Figure 5. (a, c) Cross-sectional and (b, d, f) top-view SEM pictures of $\mathrm{ZnO} / \mathrm{Alq} 3$ samples, $(\mathrm{a}, \mathrm{b})$ as produced and (c, d, f) annealed in air at $550{ }^{\circ} \mathrm{C}$. (f) High-magnification top-view SEM picture of panel d. (e) UV-vis transmittance spectra of $\mathrm{ZnO} / \mathrm{Alq} 3$ samples, as produced (gray line) or annealed at $550{ }^{\circ} \mathrm{C}$ in air (black line), and a reference spectrum of Alq3 sample grown with the same experimental parameters but without $\mathrm{ZnO}$ precursor (dotted line). 
The UV-vis transmittance spectrum is shown in Figure 5e (gray solid line) and reveals that $\mathrm{ZnO} / \mathrm{Alq} 3$ samples are transparent in the visible range, indicating that $\mathrm{Zn}$ is mainly in the oxide form $(\mathrm{ZnO})$ and not metallic. But the spectrum also shows strong absorption at wavelengths below $450 \mathrm{~nm}$. This absorption is due to the plasma polymer formed by fragmentation of Alq3 molecules. ${ }^{45-49}$ Although some organic molecules "survive" the plasma during deposition, the reactive oxygen species almost completely polymerized the organic counterpart. As a result, the absorption of Alq3 molecules located at $392 \mathrm{~nm}$ broadens significantly, and the functional properties of Alq3 molecules (i.e., fluorescence or optical absorption) are drastically suppressed due to plasma polymerization. ${ }^{45-49}$ Consequently the organic portion, mandatory for the tilted columnar nanostructuring, is not needed for the purpose of the study. Its removal was performed by annealing the samples to $550{ }^{\circ} \mathrm{C}$ in air. This treatment produces a drastic decrease in organic content of the samples (carbon and nitrogen) as revealed by EDX analysis.

Annealing of the $\mathrm{ZnO} / \mathrm{Alq} 3$ samples in air preserves the tilted nanocolumnar structure. The cross-sectional SEM image of Figure 5c shows the tilted columns, though the image is not completely clear due to charging effects. By contrast, the top-view picture of Figure $5 \mathrm{~d}$ clearly shows a highly porous structure formed by largely separated nanocolumns. Annealing of the $\mathrm{ZnO} / \mathrm{Alq} 3$ samples induces a decrease in thickness from 320 to $190 \mathrm{~nm}$ due to removal of the organic content. Besides, the annealing produces the emergence of a high amount of pores of around 10-20 nm, as observed in the high magnification image in Figure 5f. In addition to these pores, the high intrinsic porosity of GLAD thin films (usually in the range 40-50\%) due to the large intercolumnar separation indicates that the samples produced by our GAGLAD methodology are extremely porous. The UV-vis transmittance spectrum of the annealed $\mathrm{ZnO} /$ Alq3 sample is presented in Figure 5e (black solid line), where a high transparency can be noted (higher than the as-produced sample). Removal of the organic counterpart reveals the band gap of the zinc oxide, located at $380 \mathrm{~nm}$ or $3.26 \mathrm{eV}$. This $\mathrm{ZnO}$ band gap was not visible before annealing because of the absorption of the plasma polymer formed by the organic counterpart. ${ }^{45-49}$ A detailed optical study of the annealed $\mathrm{ZnO} / \mathrm{Alq} 3$ sample is shown in Figure 6. First of all, it shows a higher transmittance than the substrate (fused silica), indicating that the samples are antireflective (Figure 6a). This is produced by a smaller refractive index than the substrate (nsubs $=1.446$ ), indicating that the samples are highly porous. Unfortunately, and due to the low amount of material in a thin film, conventional adsorption isotherms cannot be accomplished. To overcome this problem, we performed an evaluation of the optical parameters that are directly related to the porosity, as will be discussed next. A simple fitting of the transmittance spectrum that takes into account the Cauchy dispersion law (see equation 1) is shown in Figure 6b, where an extraordinary agreement with the experimental spectrum can be observed. The optical values used in the fitting are displayed in the Figure $6 \mathrm{~b}$. By fixing the thickness as determined by SEM pictures $(190 \mathrm{~nm})$, the fitted refractive index turned out to be $\mathrm{n}_{0}$ (oxide) $=1.130$. The dispersion relationships of effective refractive index $\left(\mathrm{n}_{\mathrm{eff}}\right)$ and dispersion coefficient $\left(\mathrm{k}_{\text {eff }}\right)$ are presented in Figure $6 \mathrm{c}$. The small value of the refractive index $\left[\mathrm{n}_{\text {eff }}(550 \mathrm{~nm})\right.$ $=1.16]$ is accompanied by a dispersion coefficient that is particularly small for such a porous sample $\left[\mathrm{k}_{\text {eff }}(550 \mathrm{~nm})=1.1 \times 10^{-2}\right]$. 

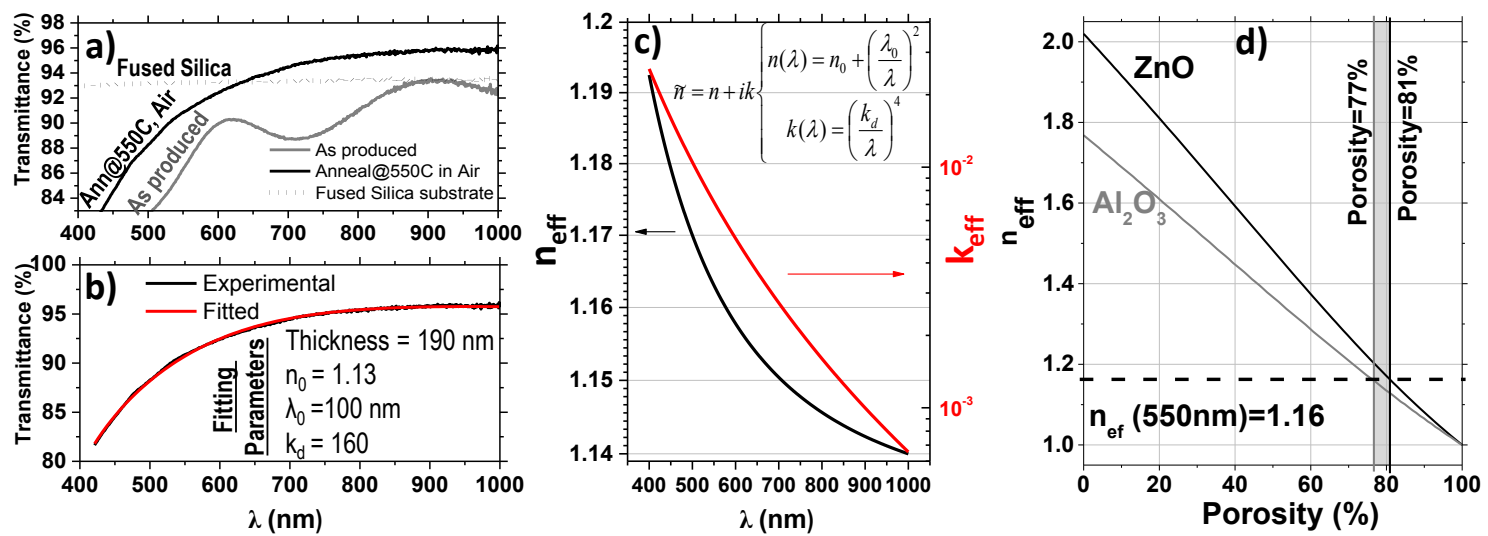

Figure 6. (a) Transmittance spectra of $\mathrm{ZnO} / \mathrm{Alq} 3$ sample, as produced (gray) and annealed at $550{ }^{\circ} \mathrm{C}$ in air (black). The spectrum of bare fused silica is also shown to visualize the increase in transmittance for the annealed sample. (b) Fitting of the transmittance spectrum of annealed $\mathrm{ZnO} / \mathrm{Alq} 3$ sample according to the Cauchy law. (c) Dispersion relationships of effective refractive index ( $\mathrm{n}_{\text {eff }}$ ) and dispersion coefficient $\left(\mathrm{k}_{\mathrm{eff}}\right)$. (d) Porosity vs effective refractive index according to Bruggeman's model for $\mathrm{ZnO}$ and $\mathrm{Al}_{2} \mathrm{O}_{3}$ with bulk refractive indexes of 2.02 and 1.77 , respectively.

Porosity can be estimated by means of effective medium modeling, like the Bruggeman method employed here. ${ }^{50}$ The refractive index of bulk $\mathrm{ZnO}$ in its most stable crystalline configuration of wurtzite is $\mathrm{n}_{\mathrm{ox}}(550 \mathrm{~nm})=2.02$. By means of the Bruggeman model, we obtain the effective refractive index ( $\left.\mathrm{n}_{\text {eff }}\right)$ as a function of porosity (see equation 2$)$. The model is shown in Figure $6 \mathrm{~d}$, where the sample with $\mathrm{n}_{\text {eff }}=1.16$ should have a porosity of $81 \%$. This point is the intersection of the fitted effective index of refraction with the curves obtained by the model. It is worth mentioning that this value is a lower limit obtained by assuming that all the pores are empty (air), although it is presumable that the pores are partially occupied by atmospheric water and therefore the samples should have even higher porosity. On the other hand, EDX analysis revealed the presence of aluminum in the films (coming from the organic molecule), ${ }^{51,52}$ indicating that a certain percentage of the sample (4 times less than $\mathrm{Zn}$ according to EDX) is aluminum oxide. But even if it is assumed that the film is entirely composed of Al2O3, the porosity estimated by the Bruggeman model is still very high, showing a minimum porosity of $77 \%$ (see lower curve in Figure 6d). It is worth mentioning that the fitting methodology of the refractive index can introduce a certain level of uncertainty, especially because of the low value and consequently the lack of interferences.

The porosity of the hybrid thin films studied here (around 80\%) is particularly high. In the methodology reported, we used Alq3 as a "sacrificial" GLAD material, whereas ZnO remains in the structure after annealing in air. The strong microstructural changes due to removal of Alq3 induce the formation of a high amount of voids, which increase the porosity of the hybrid thin films significantly. These results support that our new GAGLAD methodology can be used to obtain extremely porous $\mathrm{ZnO}$ thin films with excellent optical properties that cannot be obtained by standard GLAD. The high open porosity of the samples makes them ideal candidates for applications in fast-responsive sensors due to the high specific surface presented, which is in intimate contact with the environment. ${ }^{33}$ 


\section{CONCLUSIONS}

In this work we have developed a new technique that combines glancing angle deposition of an organic molecule with a nonglancing material of interest. We have shown that this combined codeposition technique, which we have called GAGLAD, can be used to fabricate hybrid thin films for specific applications. The anisotropic angular-selective Ag/Alq3 coatings and $\mathrm{ZnO} / \mathrm{Alq} 3$ highly porous thin films shown here have demonstrated the strength of this new methodology. The hybrid films studied are just two examples of the type of samples that can be fabricated with GAGLAD. We are convinced that the new methodology reported here will open a whole range of possibilities for the fabrication of rationally designed thin films for applications in photonics and sensors.

\section{ACKNOWLEDGMENTS}

This research was supported by the Swiss federal program Nano-Tera through the NEXRAY project.

\section{REFERENCES}

(1) Hawkeye, M. M.; Taschuk, M. T.; Brett, M. J. Glancing Angle Deposition of Thin Films: Engineering the Nanoscale; Wiley, 2014.

(2) Hawkeye, M. M.; Brett, M. J. Glancing Angle Deposition: Fabrication, Properties, and Applications of Micro- and Nanostructured Thin Films. J. Vac. Sci. Technol. A 2007, 25, 13171335.

(3) Steele, J. J.; Brett, M. J. Nanostructure Engineering in Porous Columnar Thin Films: Recent Advances. J. Mater. Sci. Mater. Electron. 2007, 18, 367-379.

(4) Sánchez-Valencia, J. R.; Borrás, A.; Barranco, A.; Rico, V. J.; Espinós, J. P.; GonzálezElipe, A. R. Preillumination of $\mathrm{TiO}_{2}$ and $\mathrm{Ta}_{2} \mathrm{O}_{5}$ Photoactive Thin Films As a Tool to Tailor the Synthesis of Composite Materials. Langmuir 2008, 24, 9460-9469.

(5) Sánchez-Valencia, J. R.; Blaszczyk-Lezak, I.; Espinós, J. P.; Hamad, S.; GonzálezElipe, A. R.; Barranco, A. Incorporation and Thermal Evolution of Rhodamine 6G Dye Molecules Adsorbed in Porous Columnar Optical $\mathrm{SiO}_{2}$ Thin Films. Langmuir 2009, 25, 9140 9148.

(6) Hamad, S.; Sánchez-Valencia, J. R.; Barranco, A.; Mejías, J. A.; González-Elipe, A. R. Molecular Dynamics Simulation of the Effect of $\mathrm{pH}$ on the Adsorption of Rhodamine Laser Dyes on $\mathrm{TiO}_{2}$ Hydroxylated Surfaces. Mol. Simul. 2009, 35, 1140-1151.

(7) Sánchez-Valencia, J. R.; Toudert, J.; González-García, L.; González-Elipe, A. R.; Barranco, A. Excitation Transfer Mechanism along the Visible to the Near-IR in Rhodamine JHeteroaggregates. Chem. Commun. 2010, 46, 4372-4374.

(8) Sánchez-Valencia, J. R.; Aparicio, F. J.; Espinós, J. P.; Gonzalez-Elipe, A. R.; Barranco, A. Rhodamine 6G and $800 \mathrm{~J}$-Heteroaggregates with Enhanced Acceptor Luminescence (HEAL) Adsorbed in Transparent $\mathrm{SiO}_{2}$ GLAD Thin Films. Phys. Chem. Chem. Phys. 2011, 13, 7071-7082.

(9) Tait, R. N.; Smy, T.; Brett, M. J. Modelling and Characterization of Columnar Growth in Evaporated Films. Thin Solid Films 1993, 226, 196-201. 
(10) Tanto, B.; Eyck, G. T.; Lu, T.-M. A Model for Column Angle Evolution during Oblique Angle Deposition. J. Appl. Phys. 2010, 108, 026107.

(11) Zhou, Q.; Li, Z.; Ni, J.; Zhang, Z. A Simple Model to Describe the Rule of Glancing Angle Deposition. Mater. Trans. 2011, 52, 469-473.

(12) Alvarez, R.; Lopez-Santos, C.; Parra-Barranco, J.; Rico, V.; Barranco, A.; Cotrino, J.; Gonzalez-Elipe, A. R.; Palmero, A. Nanocolumnar Growth of Thin Films Deposited at Oblique Angles: Beyond the Tangent Rule. J. Vac. Sci. Technol. B 2014, 32, 041802.

(13) Gish, D. A.; Nsiah, F.; McDermott, M. T.; Brett, M. J. Localized Surface Plasmon Resonance Biosensor Using Silver Nanostructures Fabricated by Glancing Angle Deposition. Anal. Chem. 2007, 79, 4228-4232.

(14) Lau, W.-F.; Bai, F.; Huang, Z. Ballistic Glancing Angle Deposition of Inclined Ag Nanorods Limited by Adatom Diffusion. Nanotechnology 2013, 24, 465707.

(15) Filippin, A. N.; Borras, A.; Rico, V. J.; Frutos, F.; González-Elipe, A. R. Laser Induced Enhancement of Dichroism in Supported Silver Nanoparticles Deposited by Evaporation at Glancing Angles. Nanotechnology 2013, 24, 045301.

(16) LaForge, J. M.; Taschuk, M. T.; Brett, M. J. Glancing Angle Deposition of Crystalline Zinc Oxide Nanorods. Thin Solid Films 2011, 519, 3530-3537.

(17) Wolcott, A.; Smith, W. A.; Kuykendall, T. R.; Zhao, Y.; Zhang, J. Z.

Photoelectrochemical Study of Nanostructured ZnO Thin Films for Hydrogen Generation from Water Splitting. Adv. Funct. Mater. 2009, 19, 1849-1856.

(18) Hrudey, P. C. P.; Westra, K. L.; Brett, M. J. Highly Ordered Organic Alq3 Chiral Luminescent Thin Films Fabricated by Glancing-Angle Deposition. Adv. Mater. 2006, 18, 224 228.

(19) Wang, Z.; Chen, Z.; Lan, Z.; Zhai, X.; Du, W.; Gong, Q. Enhancement of Alq Fluorescence by Nanotextured Silver Films Deposited on Porous Alumina Substrates. Appl. Phys. Lett. 2007, 90, 151119.

(20) Zhang, J.; Salzmann, I.; Rogaschewski, S.; Rabe, J. P.; Koch, N.; Zhang, F.; Xu, Z. Arrays of Crystalline C60 and Pentacene Nanocolumns. Appl. Phys. Lett. 2007, 90, 193117. (21) Dijken, J. G. V.; Fleischauer, M. D.; Brett, M. J. Controlled Nanostructuring of CuPc Thin Films via Glancing Angle Deposition for Idealized Organic Photovoltaic Architectures. $J$. Mater. Chem. 2011, 21, 1013-1019.

(22) Watanabe, Y.; Hyodo, S.; Motohiro, T.; Hioki, T.; Sugiura, M.; Noda, S. Catalytic Properties of Thin Films by Simultaneous Oblique Sputter Deposition of Two Materials from Different Directions. Thin Solid Films 1995, 256, 68-72.

(23) He, Y.; Wu, J.; Zhao, Y. Designing Catalytic Nanomotors by Dynamic Shadowing Growth. Nano Lett. 2007, 7, 1369-1375.

(24) Tesfamichael, T.; Motta, N.; Bostrom, T.; Bell, J. M. Development of Porous Metal Oxide Thin Films by Co-Evaporation. Appl. Surf. Sci. 2007, 253, 4853-4859.

(25) He, Y.; Zhang, Z.; Hoffmann, C.; Zhao, Y. Embedding Ag Nanoparticles into $\mathrm{MgF}_{2}$ Nanorod Arrays. Adv. Funct. Mater. 2008, 18, 1676-1684.

(26) Vauth, S.; Streng, C.; Mayr, S.; Samwer, K. Growth of Vapor-Deposited Amorphous Zr65A17.5Cu27.5 Films under Oblique Particle Incidence Investigated by Experiment and Simulation. Phys. Rev. B 2003, 68 .

(27) Su, X.; Li, M.; Zhou, Z.; Zhai, Y.; Fu, Q.; Huang, C.; Song, H.; Hao, Z. Microstructure and Multiphoton Luminescence of Au Nanocrystals Prepared by Using Glancing Deposition Method. J. Lumin. 2008, 128, 642-646. 
(28) He, Y.; Zhao, Y.; Wu, J. The Effect of Ti Doping on the Growth of Mg Nanostructures by Oblique Angle Codeposition. Appl. Phys. Lett. 2008, 92, 063107.

(29) Zhou, C.; Gall, D. Two-Component Nanorod Arrays by Glancing-Angle Deposition. Small 2008, 4, 1351-1354.

(30) Smith, W.; Zhao, Y. Enhanced Photocatalytic Activity by Aligned $\mathrm{WO}_{3} / \mathrm{TiO}_{2}$ TwoLayer Nanorod Arrays. J. Phys. Chem. C 2008, 112, 19635-19641.

(31) He, Y.; Fu, J.; Zhang, Y.; Zhao, Y.; Zhang, L.; Xia, A.; Cai, J. Multilayered Si/Ni Nanosprings and Their Magnetic Properties. Small 2007, 3, 153-160.

(32) Kesapragada, S. V.; Gall, D. Two-Component Nanopillar Arrays Grown by Glancing Angle Deposition. Thin Solid Films 2006, 494, 234-239.

(33) Sanchez-Valencia, J. R.; Alcaire, M.; Romero-Gómez, P.; Macias-Montero, M.; Aparicio, F. J.; Borras, A.; Gonzalez-Elipe, A. R.; Barranco, A. Oxygen Optical Sensing in Gas and Liquids with Nanostructured ZnO Thin Films Based on Exciton Emission Detection. $J$. Phys. Chem. C 2014, 118, 9852-9859.

(34) Gonzalez-García, L.; Parra-Barranco, J.; Sanchez-Valencia, J. R.; Ferrer, J.; GarciaGutierrez, M.-C.; Barranco, A.; Gonzalez-Elipe, A. R. Tuning Dichroic Plasmon Resonance Modes of Gold Nanoparticles in Optical Thin Films. Adv. Funct. Mater. 2013, 23, 1655-1663. (35) Sanchez-Valencia, J. R.; Toudert, J.; Borras, A.; Barranco, A.; Lahoz, R.; de la Fuente, G. F.; Frutos, F.; Gonzalez-Elipe, A. R. Selective Dichroic Patterning by Nanosecond Laser Treatment of Ag Nanostripes. Adv. Mater. 2011, 23, 848-853.

(36) Sánchez-Valencia, J. R.; Toudert, J.; Borras, A.; López-Santos, C.; Barranco, A.; Feliu, I. O.; González-Elipe, A. R. Tunable In-Plane Optical Anisotropy of Ag Nanoparticles Deposited by DC Sputtering onto $\mathrm{SiO}_{2}$ Nanocolumnar Films. Plasmonics 2010, 5, 241-250.

(37) Parra-Barranco, J.; García-García, F. J.; Rico, V.; Borrás, A.; López-Santos, C.; Frutos, F.; Barranco, A.; González-Elipe, A. R. Anisotropic In-Plane Conductivity and Dichroic Gold Plasmon Resonance in Plasma-Assisted ITO Thin Films E-Beam-Evaporated at Oblique Angles. ACS Appl. Mater. Interfaces 2015, 7, 10993-11001.

(38) Ditchburn, R. J.; Smith, G. B. Useful Angular Selectivity in Oblique Columnar Aluminum. J. Appl. Phys. 1991, 69, 3769-3771.

(39) Mbise, G. W.; Bellac, D. L.; Niklasson, G. A.; Granqvist, C. G. Angular Selective Window Coatings: Theory and Experiments. J. Phys. Appl. Phys. 1997, 30, 2103-2122.

(40) Granqvist, C. G. Materials Science for Solar Energy Conversion Systems; Elsevier, 2013.

(41) Höhn, O.; Peters, M.; Ulbrich, C.; Hoffmann, A.; Schwarz, U. T.; Bläsi, B. Optimization of Angularly Selective Photonic Filters for Concentrator Photovoltaic. Proc. SPIE 2012, 8438,84380 A - 84380A.

(42) Bermel, P.; Ghebrebrhan, M.; Chan, W.; Yeng, Y. X.; Araghchini, M.; Hamam, R.; Marton, C. H.; Jensen, K. F.; Soljačić, M.; Joannopoulos, J. D.; Johnson, S. G.; Celanovic, I. Design and Global Optimization of High-Efficiency Thermophotovoltaic Systems. Opt. Express 2010, 18, A314-A334.

(43) MacMASTER, S. W. Privacy Screen for a Display. Patent US7052746 B2, May 30, 2006.

(44) Shen, Y.; Ye, D.; Celanovic, I.; Johnson, S. G.; Joannopoulos, J. D.; Soljačić, M. Optical Broadband Angular Selectivity. Science 2014, 343, 1499-1501.

(45) Aparicio, F. J.; Borras, A.; Blaszczyk-Lezak, I.; Gröning, P.; Álvarez-Herrero, A.; Fernández-Rodríguez, M.; González-Elipe, A. R.; Barranco, A. Luminescent and Optical 
Properties of Nanocomposite Thin Films Deposited by Remote Plasma Polymerization of Rhodamine 6G. Plasma Process. Polym. 2009, 6, 17-26.

(46) Aparicio, F. J.; Blaszczyk-Lezak, I.; Sánchez-Valencia, J. R.; Alcaire, M.; González, J. C.; Serra, C.; González-Elipe, A. R.; Barranco, A. Plasma Deposition of Perylene-Adamantane Nanocomposite Thin Films for $\mathrm{NO}_{2}$ Room-Temperature Optical Sensing. J. Phys. Chem. C 2012, 116, 8731-8740.

(47) Blaszczyk-Lezak, I.; Aparicio, F. J.; Borrás, A.; Barranco, A.; Álvarez-Herrero, A.; Fernández-Rodríguez, M.; González-Elipe, A. R. Optically Active Luminescent Perylene Thin Films Deposited by Plasma Polymerization. J. Phys. Chem. C 2008, 113, 431-438.

(48) Barranco, A.; Aparicio, F.; Yanguas-Gil, A.; Groening, P.; Cotrino, J.; González-Elipe, A. R. Optically Active Thin Films Deposited by Plasma Polymerization of Dye Molecules. Chem. Vap. Depos. 2007, 13, 319-325.

(49) Aparicio, F. J.; Alcaire, M.; Borras, A.; Gonzalez, J. C.; López-Arbeloa, F.; BlaszczykLezak, I.; González-Elipe, A. R.; Barranco, A. Luminescent 3-Hydroxyflavone Nanocomposites with a Tuneable Refractive Index for Photonics and UV Detection by Plasma Assisted Vacuum Deposition. J. Mater. Chem. C 2014, 2, 6561-6573.

(50) Stroud, D. The Effective Medium Approximations: Some Recent Developments. Superlattices Microstruct. 1998, 23, 567-573.

(51) Alcaire, M.; Sanchez-Valencia, J. R.; Aparicio, F. J.; Saghi, Z.; Gonzalez-Gonzalez, J. C.; Barranco, A.; Zian, Y. O.; Gonzalez-Elipe, A. R.; Midgley, P.; Espinos, J. P.; Groening, P.; Borras, A. Soft Plasma Processing of Organic Nanowires: A Route for the Fabrication of 1D Organic Heterostructures and the Template Synthesis of Inorganic 1D Nanostructures.

Nanoscale 2011, 3, 4554-4559.

(52) Alcaire, M.; Filippin, A. N.; Macias-Montero, M.; Sanchez-Valencia, J. R.; Rojas, T. C.; Mora-Boza, A.; Lopez-Santos, C.; Espinos, J. P.; Barranco, A.; Borras, A. Highly Porous ZnO Thin Films and 1D Nanostructures by Remote Plasma Processing of Zn-Phthalocyanine. Plasma Process. Polym. 2016, 13, 287-297.

\section{ASSOCIATED CONTENT}

Supporting Information Available.

Three figures showing cross-sectional SEM image of Ag sample in absence of organic molecules (S1), fluorescence spectra for Ag/Alq3 samples (S2), and full sequence of transmittance spectra for $\mathrm{x}$ - and $\mathrm{y}$-rotations and polarizations (S3).

Movie S1, a composition of the 70 ADF-STEM images taken at $2^{\circ}$ tilt intervals (AVI)

Movie S2, series of orthoslices through the 3D tomographic reconstruction (AVI) 


\section{Supporting Information}

\section{Growth Assisted by Glancing Angle Deposition: A New Technique to Fabricate Highly Porous Anisotropic Thin Films}

Juan Ramon Sanchez-Valencia, ${ }^{*}+\perp$ Remi Longtin, ${ }^{*}$ Marta D. Rossell, ${ }^{\S}$ and Pierangelo Gröning"

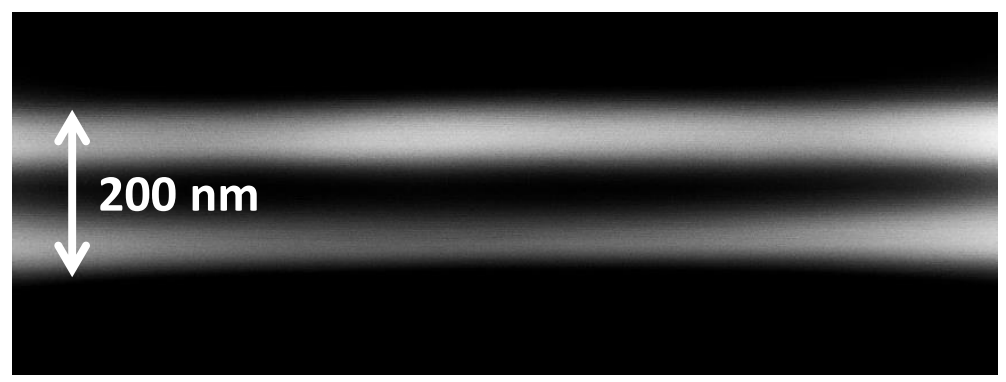

Supporting Information S1. Cross-sectional SEM image of silver sample (with the highest amount of silver) grown in absence of organic molecules. The picture shows an equivalent Ag thickness of $200 \mathrm{~nm}$

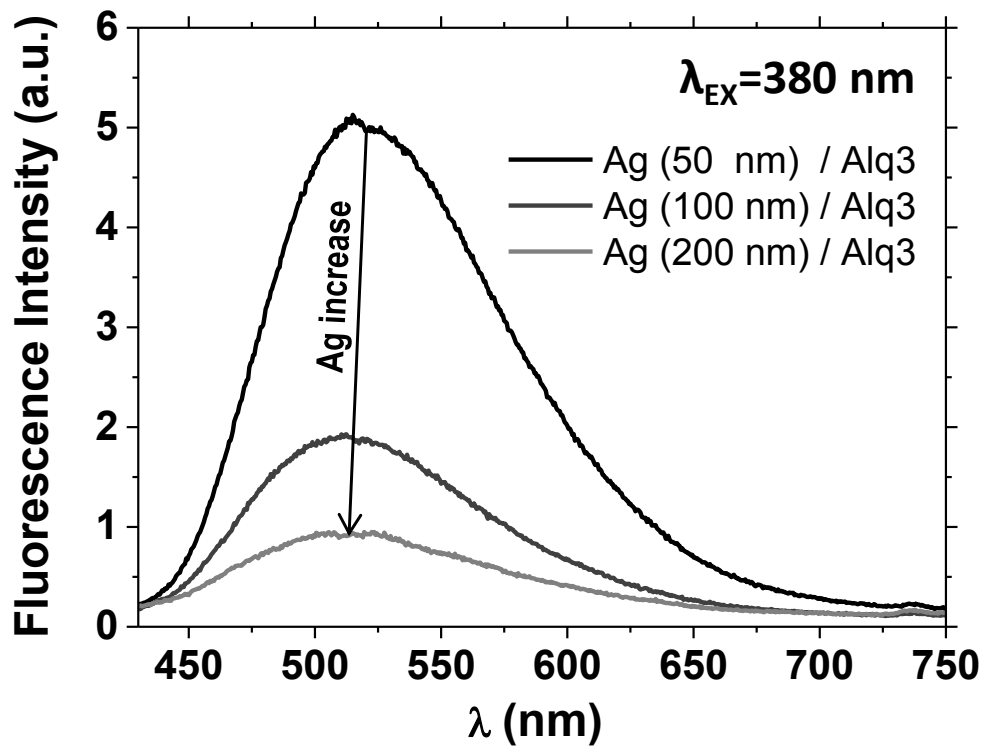

Supporting Information S2. Fluorescence spectra of Ag/Alq3 thin films with 50, 100 and $200 \mathrm{~nm}$ of Ag equivalent thickness. It can be observed a gradual decrease of the fluorescence intensity with the $\mathrm{Ag}$ thickness. 
$\operatorname{Ag}(50 \mathrm{~nm}) / \mathrm{Alq3}$

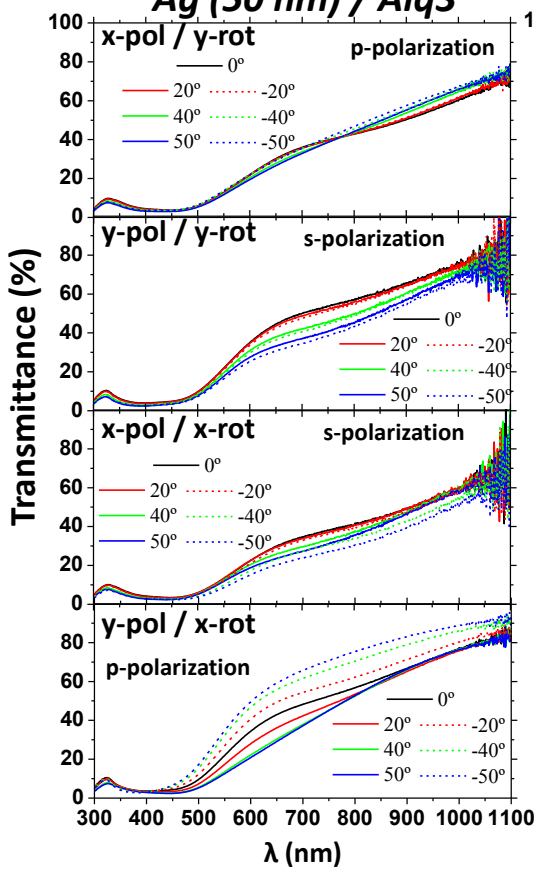

$\operatorname{Ag}(100 \mathrm{~nm}) / \mathrm{Alq3}$
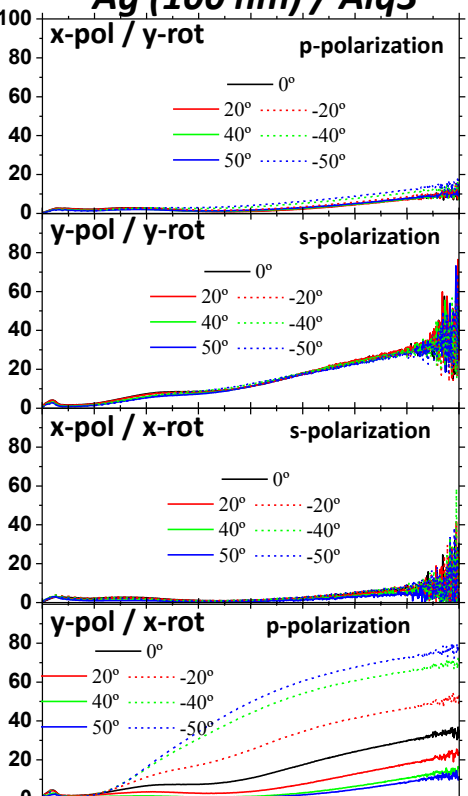

$\operatorname{Ag}(200 \mathrm{~nm}) / \mathrm{Alq3}$

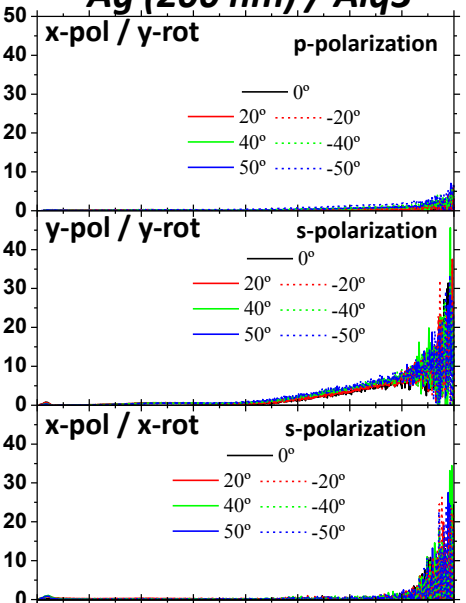

y-pol / x-rot p-polarization

$30-\frac{1}{20^{\circ}} 0^{\circ}$

$-40^{\circ} \cdots \cdots-20^{\circ}$
$-50^{\circ}$

Supporting Information S3. Transmittance spectra set for $\mathrm{x}$ - and $\mathrm{y}$ - polarizations and rotations around $\mathrm{x}$ and y axes, for Ag/Alq3 thin films with 50 (left), 100 (middle) and $200 \mathrm{~nm}$ (right) of Ag equivalent thickness (samples in Fig 2 and 4). Samples were rotated from $-50^{\circ}$ (dashed lines) to $+50^{\circ}$ (solid lines). 